\title{
PPAR- $\gamma 2$ Pro12Ala Variant, Insulin Resistance and Plasma Long-chain Polyunsaturated Fatty Acids in Childhood Obesity
}

\author{
SILVIA SCAGLIONI, ELVIRA VERDUCI, MICHELA SALVIONI, MARIA LUISA BIONDI, GIOVANNI RADAELLI, \\ CARLO AGOSTONI, AND MARCELLO GIOVANNINI \\ Department of Pediatrics [S.S., E.V., M.S., C.A., M.G.], Unit of Medical Statistics [G.R.], Department of Clinical Chemistry and \\ Microbiology [M.L.B.], San Paolo Hospital; University of Milan, I-20142 Milan, Italy
}

\begin{abstract}
Pro12Ala variant of peroxisome-proliferator-activated receptor-gamma2 (PPAR- $\gamma 2$ ) may be linked to insulin sensitivity. This study examined whether an association of PPAR- $\gamma 2$ Pro12Ala with insulin resistance and plasma LCPUFAs may exist in obese children. One hundred and forty Italian normolipidemic obese children (58 girls and 82 boys, mean age [SD], 10.2 [2.7] y) entered the study. Obesity was defined according to International Obesity Task Force. BMI Z-scores were calculated. Fasting blood glucose, insulin, lipids and plasma fatty acids were measured. Insulin resistance was estimated by the homeostatic model assessment (HOMA-IR). The frequency of Ala allele was 9\%. Mean [SD] values of fasting insulin and HOMA-IR in Pro/Pro versus Pro12Ala groups were: 19.3 [10.6] versus $14.1[10.4] \mu \mathrm{U} / \mathrm{mL}(p=0.017)$ and 4.2 [2.3] versus 3.0 [2.3] $(p=0.022)$. Mean $[\mathrm{SD}]$ values of plasma C20:3n-9 and of C20:4n-6, C20:5n-3, C22:6n-3 and n-6/n-3 LCPUFA in phospholipds in Pro/ Pro versus Pro12Ala groups were: 0.15 [0.07] versus 0.12 [0.08] \% $(p=0.014), 8.9$ [1.9] versus $10.2[2.6] \%(p=0.023), 0.34[0.15]$ versus $0.42[0.11] \%(p=0.005), 2.1[0.9]$ versus $2.6[0.9] \%(p=$ $0.032)$ and $4.8[1.2]$ versus $4.2[0.7](p=0.017)$. Pro12Ala may be associated with higher insulin sensitivity and higher LCPUFAs, particularly n-3, levels in plasma phosholipids of obese children.
\end{abstract} (Pediatr Res 60: 485-489, 2006)

$\mathrm{C}$ hildhood obesity is an increasing condition in the general population, possibly associated with later onset of chronic disturbances and clinical complications, such as type 2 diabetes mellitus and cardiovascular disease (1), and represents an emerging health problem (2). Insulin resistance, glucose intolerance and hyperinsulinemia are major components of the metabolic syndrome (3), highly prevalent among children and adolescents with marked obesity (4). Although the mechanisms underlying insulin resistance are not fully elucidated, both genetic factors and environmental factors leading to obesity play relevant roles in its etiology (5).

Among genetic factors, peroxisome-proliferator-activated receptor-gamma (PPAR- $\gamma 2$ ), a nuclear transcription factor, is involved in the metabolic disposal of the adipose tissue (6) including adipocyte differentiation, adipogenesis and the pathogenesis of insulin resistance states (7-9). The Pro12Ala variant of PPAR- $\gamma 2$, highly prevalent in Caucasians (10), has

Received February 24, 2006; accepted May 30, 2006

Correspondence: Silvia Scaglioni, M.D., Department of Pediatrics, San Paolo Hospital, University of Milan, Via A di Rudinì 8, I-20142 Milan, Italy; E-mail: silvia.scaglioni@unimi.it

DOI: $10.1203 / 01 . p d r .0000238259 .41560 .00$ been studied for the association with obesity-related or insulin resistance-related traits (11-13). Some studies suggest that the Pro12Ala genotype may be associated with improved insulin sensitivity $(12,13)$, especially in obese subjects, but this matter is still controversial (14-16).

Among environmental factors, the quality of dietary fats may be also linked to the metabolic syndrome, particularly in adults, with excess saturated fats enhancing, and unsaturated fats preventing, the development of the major complications of the metabolic syndrome through several independent mechanisms including the blood lipid picture and other mechanisms linked to the atherogenic process (http://www.healthierus.gov/ dietaryguidelines/).

Within this context, polyunsaturated fatty acids (PUFAs) are able to activate PPAR $\gamma$, leading to a decreased insulin resistance status (17). PUFA-enriched diets (such as the Eskimo diet) have been associated with increased insulin sensitivity and decreased triglyceride levels in rodents and humans (18), two effects that are shared by the synthetic PPAR $\gamma$ agonists. Also the main PUFA derivatives, arachidonic and docosahexanaenoic acids, are effective PPAR activators (19).

Despite the potential clinical relevance of the association between Pro12Ala variant of PPAR- $\gamma 2$ and insulin resistance with the long-chain polyunsaturated fatty acid (LCPUFA) status, there is lack of studies in the pediatric population. Aim of the present study was to examine whether an association of PPAR- $\gamma 2$ Pro12Ala variant might exist with measures of insulin resistance and the plasma LCPUFA profile in obese white children.

\section{MATERIALS AND METHODS}

The present observational study included 140 obese children (mean age [SD], 10.2 [2.7] y, 58 girls and 82 boys), consecutively admitted to our department from January, 2003 to October, 2005.

Inclusion criteria were: weight at birth $\geq 2500 \mathrm{~g}$, gestational age $37-42 \mathrm{wk}$ inclusive, singleton birth, obesity, and having white parents. Children exhibiting diabetes mellitus and/or endocrine diseases, chronic liver diseases, and infectious disorders were excluded. Additionally, children having hyperlipidemia according to Italian guidelines (20) were excluded to prevent possible bias effects on plasma fatty acid status.

Abbreviations: FA, fatty acid; HOMA-IR, homeostatic model assessment; LCPUFA, long chain polyunsaturated fatty acid; PL, phospholipids; PPAR$\boldsymbol{\gamma} \mathbf{2}$, peroxisome-proliferator-activated receptor-gamma; PUFAs, polyunsaturated fatty acids 
All children were born in Lombardy, central-northern Italy, where they are currently residing. A pediatrician described the investigation and written informed consent was obtained from both parents. The Institutional Ethical Committee approved the study.

A medical history was obtained from parents by a standardized questionnaire at a personal interview conducted by the same pediatrician before the child's hospital discharge. Anthropometrics (height, weight), blood pressure, fasting biochemical features (glucose, insulin levels, lipid and fatty acids profile) of children were evaluated during the hospital stay, within three days of recruitment. The same pediatrician performed the Tanner staging of puberty.

Two experienced pediatricians, according to standardized procedures, performed anthropometric measurements. The body weight $(\mathrm{kg})$ was measured with an electronic scale accurate to $0.1 \mathrm{~kg}$ and height $(\mathrm{cm})$ was measured to the nearest $0.5 \mathrm{~cm}$ with a Harpenden stadiometer. For each anthropometric variable, three measurements were taken and the mean value was considered for the analysis. The coefficient of variation of the measurements ranged from $0.8-1.4 \%$ (observer 1) and from $0.7-1.4 \%$ (observer 2). This small and comparable degree of inter-observers measurement variation was deemed to yield reliable anthropometric data. The body mass index (BMI) was calculated from the ratio of weight to length/height ${ }^{2}\left(\mathrm{~kg} / \mathrm{m}^{2}\right)$. In accordance with the International Obesity Task Force, a child was defined obese if her/his BMI was above the age- and sex-adjusted BMI Cole's curve passing through the cut-off of $30 \mathrm{~kg} / \mathrm{m}^{2}$ at $18 \mathrm{y}$ (21). Compared with Italian age- and sex-adjusted growth charts for BMI (22), all recruited eligible children exhibited BMI over $97^{\text {th }}$ percentile. BMI Z-scores were calculated and adjusted for age and gender by using the LMS-method of Cole (23) and Italian reference data (22).

At the hospital, parents had body weight and height measured. A parent was defined overweight if her/his BMI was equal or greater than $25 \mathrm{~kg} / \mathrm{m}^{2}$, obese if BMI was equal or greater than $30 \mathrm{~kg} / \mathrm{m}^{2}$. Parental overweight/obesity was defined when at least one parent was overweight/obese.

Blood samples were collected after overnight fasting. Serum insulin levels $(\mu \mathrm{U} / \mathrm{mL})$ were determined by a electrochemiluminescence immune assay analyzer, and plasma glucose levels $(\mathrm{mg} / \mathrm{dL}$ ) by enzymatic method. Flavored glucose in a dose of $1.75 \mathrm{~g} / \mathrm{kg}$ of body weight (up to a maximum of $75 \mathrm{~g}$ ) was given orally, and blood samples were obtained for measurements of plasma glucose and serum insulin at baseline and plasma glucose at $120 \mathrm{~min}$. Insulin resistance was estimated by the homeostatic model assessment (HOMA-IR) (24). HOMA-IR is calculated as the product of fasting glucose ( $\mathrm{mmol} / \mathrm{L})$ and fasting insulin $(\mu \mathrm{U} / \mathrm{mL})$, divided by 22.5 .

Total cholesterol (TC) and triacylglycerol (TG) plasma levels were measured using a dry multiplayer enzymatic method (Ectachem DT-60; Eastman Kodak Co., Rochester, NY). LDL-cholesterol (LDL-C) serum levels were calculated according to the Friedewald formula (LDL-C $=$ TC $-[$ HDL-C + TG/5]) (25). Plasma fatty acid (FA) analyses (expressed as \% total FA) were performed by means of capillary gas chromatography after lipid extraction according to Folch et al. (26) and the phospholipid fraction of plasma fatty acids has been separated by thin-layer chromatography as described (27).

Genetic assessment was based on 200 base pairs of sequence surrounding PPAR 22 Pro12Ala, provided to Applied Biosystems (Foster City, CA) to develop Taqman Allelic Dicrimination (AD) Assays using their assay by design platform. Genotyping of the Pro12Ala AD was performed using primers $\left(0.9 \mu \mathrm{moli} / \mathrm{L}\right.$ each). Forward $5^{\prime}$-TTATGGGTGAAACTCTGGGAGATT-3' and reverse 5' $^{\prime}$-TGCAGACAGTGTATCAGTGAAGGA-3' and the Taqman MGB probes Fam-TTCTGGGTCAATAGG and Vic-CTTTCTGCGTCAATAG $(0.1 \mu \mathrm{moli} / \mathrm{L}$ each; Applied Biosystems). Four microliters of a $10 \mathrm{ng} / \mu \mathrm{L}$ stock of DNA was dispensed into 384-well PCR plates using a Biomek FX robot (Beckman Coulter, Fullerton, CA, USA), to wich $6 \mu \mathrm{L}$ of a mix containing primers, MGB probes, and TaqMan Universal PCR Master Mix (Applied Biosystems) and incubated at $95^{\circ} \mathrm{C}$ for 10 min followed by 40 cycles of $95^{\circ} \mathrm{C}$ for $15 \mathrm{~s}$ and $60^{\circ} \mathrm{C}$ for $1 \mathrm{~min}$ before analysis on a $7900 \mathrm{HT}$ plate reader (Applied Biosystems). Individual genotypes were determined using SDSv2.1 software (Applied Biosystems).

The same experienced radiologist performed liver untrasonography by a Hitachi H21 (Hitachi High Technology Corporation Ltd., Tokio, Japan) using a 3.5-MHz convex array transducer. Longitudinal, subcostal, ascending, and oblique scans were performed (28). Liver echogenicity was evaluated independently on videotape by three radiologists unaware of the subject, and a consensus was established. Absolute agreement among judgements before discussion occurred in 134/140 (95.7\%) cases.

Finally, dietary habits of children were assessed at recruitment by means of an age-adjusted Food Frequency Questionnaire made up of 116 items and designed according to Block (29). The same experienced dietician, unaware of the obesity status of children, interviewed mothers for approximately 50 min and each meal was analyzed to find out which food was eaten and how often. Usual portion sizes were estimated using household measures, the weight of purchase (e.g., pasta) or unit (e.g., fruit juice). A 24-h recall on the day before the hospital admission was further recorded at the end of the interview to standardize the usual serving size. Quantification and analysis of the energy intake and nutrient composition were performed with an ad hoc PC software program developed at our department and based on the Food and Nutrient Data Base issued by the National Institute of Nutrition (30).

Statistical analysis. Descriptive data are shown as mean (SD) or number of observations (percentage). The $\chi^{2}$ test was used for comparing discrete variables. Comparison between groups for continuous variables was performed by the $t$ test for unpaired data or the Mann-Whitney test, as appropriate. Triglycerides, insulin and HOMA-IR were not normally distributed and $\log _{10}$-transformation was used for analysis. For clarity of interpretation, results are expressed as untransformed values. Adjustments were made for potential confounders (infant's gender, age, body weight at birth, Tanner stage, BMI Z-score, duration of obesity and BMI* PPAR $\gamma 2$ interaction. The association of the Pro12Ala polymorphism with indices of insulin resistance (fasting insulin levels, HOMA-IR) and plasma FA was tested using multiple linear regression. All $p$-values $<0.05$ were considered to indicate statistical significance (two-tailed test).

\section{RESULTS}

The frequency of Ala allele was 9\% (Pro/Pro 81.4\%, Pro/ Ala 18.6\%). No child was carrier of Ala/Ala genotype. Table 1 reports clinical and biochemical variables of children according to Pro12Ala PPAR- $\gamma 2$ genotype. Ala carriers, compared with non-carriers, showed lower fasting insulin levels $(p$ $<0.05)$ and lower HOMA-IR $(p<0.05)$. These association remained significant (fasting insulin, odds ratio [OR] 0.94, 95\% confidence interval [CI] 0.88-1.00; HOMA-IR, OR 0.74, 95\% CI 0.55-0.99) also at a multiple logistic regression analysis fitted for PPAR $\gamma 2$ and including variables listed in Table 1 as covariates. Pro12Ala genotype was not associated with any other cardiovascular traits listed in Table 1 both before (Table 1) and after adjusting for either fasting insulin or HOMA-IR (any $p>0.413$ ). No significant interaction effect

Table 1. Clinical and biochemical parameters of children according to Pro12Ala PPAR- $\gamma 2$ genotype

\begin{tabular}{lccc}
\hline \multicolumn{1}{c}{ Characteristic } & $\begin{array}{c}\text { Pro/Pro } \\
(\mathrm{N}=114)\end{array}$ & $\begin{array}{c}\text { Pro/Ala } \\
(\mathrm{N}=26)\end{array}$ & $p$-value $\dagger$ \\
\hline Gender (boys/girls) & $64 / 50$ & $18 / 8$ & 0.336 \\
Age (y) & $10.2 \pm 2.7$ & $10.0 \pm 2.7$ & 0.743 \\
BMI Z-score & $2.23 \pm 0.43$ & $2.30 \pm 0.32$ & 0.436 \\
Duration of obesity (y) & $4.9 \pm 2.5$ & $5.1 \pm 2.4$ & 0.466 \\
Systolic BP (mm Hg) & $113 \pm 10$ & $116 \pm 10$ & 0.143 \\
Diastolic BP (mm Hg) & $69 \pm 7$ & $70 \pm 9$ & 0.670 \\
Fasting glucose (mmol/L) & $4.81 \pm 0.32$ & $4.75 \pm 0.32$ & 0.620 \\
120min OGTT glucose & $6.15 \pm 1.26$ & $6.16 \pm 0.89$ & 0.792 \\
$\quad(m m o l / L)$ & & & \\
Fasting insulin (mU/mL) & $19.3 \pm 10.5$ & $14.1 \pm 10.4$ & $0.024^{*}$ \\
HOMA-IR & $4.2 \pm 2.3$ & $3.0 \pm 2.3$ & $0.040^{*}$ \\
Total cholesterol (mmol/L) & $4.19 \pm 0.67$ & $4.35 \pm 0.65$ & 0.288 \\
HDL cholesterol (mmol/L) & $1.29 \pm 0.27$ & $1.29 \pm 0.28$ & 0.994 \\
LDL cholesterol (mmol/L) & $2.35 \pm 0.63$ & $2.46 \pm 0.55$ & 0.430 \\
Triglycerides (mmol/L) & $1.14 \pm 0.64$ & $1.29 \pm 0.83$ & 0.470 \\
Increased liver echogenicity & $18 / 96$ & $6 / 20$ & 0.375 \\
$\quad(y e s / n o)$ & & & \\
\hline
\end{tabular}

Values are mean \pm SD or number of observations.

* Statistically significant.

$\dagger P$-values were adjusted for infant's gender, age, body weight at birth, Tanner stage, BMI Z-score, duration of obesity and BMI* PPAR $\gamma 2$ interaction.

SI convertion factors: to convert glucose to $\mathrm{mg} / \mathrm{dL}$, divide values by 0.0555 ; to convert cholesterol, divide values by 0.0259 ; to convert triglycerides, divide values by 0.0113 . 
was observed between BMI and Pro12Ala polymorphism in modulating insulin sensitivity (HOMA- IR $p$-value interaction $=0.480$; fasting insulin $p$-value interaction $=0.396$ ).

Increased liver echogenicity was found in 24 out of 140 cases $(17 \%)$. The distribution of Tanner stage did not differ $(p$ $=0.655$ ) between carriers and noncarriers: stage I, $46.2 \%$ versus $42.7 \%$; stage II-III, $38.5 \%$ versus $33.6 \%$; stage IV-V, $15.4 \%$ versus $23.6 \%$, respectively. Familial overweight was found in $38.5 \%$ and $36.8 \%$ of Ala carriers and noncarriers, respectively ( $p=0.997)$, familial obesity was $40.4 \%$ versus $53.8 \%(p=0.273)$.

No significant differences were found between Ala carriers and non-carriers for total dietary energy or any macronutrients intake: mean (SD) total energy ( $\mathrm{kcal} / \mathrm{die}), 2467$ (910) versus $2765(935)(p=0.188)$; protein $(\%), 15(2)$ versus 15 (2) $(p$ $=0.487)$; carbohydrate $(\%), 57$ (7) versus $56(6)(p=0.165)$; fat (\%), 31 (5) versus $32(5)(p=0.433)$. No inter-group differences were found with respect to the dietary intake of the main FA families, including PUFAs (\%), 4.1 (0.5) versus 4.2 (0.5) $(p=0.327)$, or polyunsaturated to saturated fatty acid ratio, 0.39 (0.11) versus $0.39(0.10)(p=0.391)$.

Table 2 shows the relationship of the plasma FA profile of total lipids with Pro12Ala PPAR- $\gamma 2$ genotype. The Pro12Ala polymorphism was associated with lower plasma C20:3n-9 ( $p$ $<0.05$ ). The FA composition of plasma phospholipids (PL) according to PPAR- $\gamma 2$ genotype is reported in Table 3. The Pro12Ala polymorphism was significantly associated with lower plasma PL n-6/n-3 PUFA, n-6/n-3 LCPUFA and higher levels of n-3 PUFA, n-6 LCPUFA, n-3 LCPUFA, C20:4n-6, C20:5n-3 and C22:6n-3.

A logistic regression model was further fitted for PPAR $\gamma 2$ genotype, and including HOMA-IR and \% plasma phospholipids and confounders as covariates, to assess the independent association of Pro12Ala with LCPUFA and HOMA-IR. This analysis showed that phospholipid n-6/n-3 PUFA was the only

Table 2. LCPUFA as FA \% of plasma total lipids according to Prol2Ala PPAR- $\gamma 2$ genotype

\begin{tabular}{lccc}
\hline \multicolumn{1}{c}{ Variable } & Pro/Pro & Pro/Ala & \\
\hline Saturated FA & $(\mathrm{N}=114)$ & $(\mathrm{N}=26)$ & $p$-value \\
Monounsaturated FA & $35.9 \pm 2.6$ & $36.5 \pm 2.6$ & 0.069 \\
Polyunsaturated FA & $26.4 \pm 3.3$ & $26.4 \pm 2.2$ & 0.566 \\
n-6 PUFA & $37.6 \pm 4.7$ & $37.0 \pm 3.3$ & 0.496 \\
n-3 PUFA & $34.8 \pm 4.5$ & $34.2 \pm 3.1$ & 0.397 \\
n-6/n-3 PUFA & $2.6 \pm 0.7$ & $2.7 \pm 0.5$ & 0.125 \\
n-6 LCPUFA & $13.9 \pm 3.3$ & $12.9 \pm 2.1$ & 0.132 \\
n-3 LCPUFA & $10.0 \pm 1.6$ & $10.4 \pm 1.4$ & 0.243 \\
n-6/n-3 LCPUFA & $2.3 \pm 0.7$ & $2.4 \pm 0.4$ & 0.092 \\
C18:2n-6 & $4.5 \pm 1.0$ & $4.3 \pm 0.7$ & 0.513 \\
C20:4n-6 & $24.7 \pm 4.2$ & $23.8 \pm 2.6$ & 0.156 \\
C18:3n-3 & $7.2 \pm 1.3$ & $7.3 \pm 1.3$ & 0.822 \\
C20:5n-3 & $0.29 \pm 0.10$ & $0.27 \pm 0.08$ & 0.668 \\
C22:6n-3 & $0.44 \pm 0.19$ & $0.44 \pm 0.11$ & 0.446 \\
C20:3n-9 & $1.6 \pm 0.6$ & $1.6 \pm 0.4$ & 0.274 \\
C20:5n-3/C20:4n-6 & $0.15 \pm 0.07$ & $0.12 \pm 0.08$ & $0.014 *$ \\
C20:3n-9/ C20:4n-6 & $0.022 \pm 0.016$ & $0.018 \pm 0.013$ & 0.061 \\
C22:6n-3/C20:4n-6 & $0.21 \pm 0.06$ & $0.22 \pm 0.04$ & 0.402 \\
\hline
\end{tabular}

Values are mean $\pm \mathrm{SD}$.

* Statistically significant.
Table 3. LCPUFA as FA \% of plasma phospholipids according to Pro12Ala PPAR- $\gamma 2$ genotype

\begin{tabular}{lccl}
\hline \multicolumn{1}{c}{ Variable } & $\begin{array}{c}\text { Pro/Pro } \\
(\mathrm{N}=114)\end{array}$ & $\begin{array}{c}\text { Pro/Ala } \\
(\mathrm{N}=26)\end{array}$ & $p$-value \\
\hline Saturated FA & $48.3 \pm 2.7$ & $46.7 \pm 6.1$ & 0.700 \\
Monounsaturated FA & $16.9 \pm 2.3$ & $16.6 \pm 2.5$ & 0.250 \\
Polyunsaturated FA & $34.7 \pm 3.5$ & $36.6 \pm 5.5$ & 0.340 \\
n-6 PUFA & $31.5 \pm 2.8$ & $32.7 \pm 4.6$ & 0.415 \\
n-3 PUFA & $3.1 \pm 1.1$ & $3.7 \pm 1.1$ & $0.006^{*}$ \\
n-6/n-3 PUFA & $11.6 \pm 4.2$ & $9.0 \pm 1.3$ & $0.001^{*}$ \\
n-6 LCPUFA & $13.1 \pm 2.5$ & $15.0 \pm 2.9$ & $0.002^{*}$ \\
n-3 LCPUFA & $2.9 \pm 1.1$ & $3.6 \pm 1.1$ & $0.004^{*}$ \\
n-6/n-3 LCPUFA & $4.8 \pm 1.2$ & $4.2 \pm 0.7$ & $0.017^{*}$ \\
C18:2n-6 & $18.4 \pm 2.3$ & $17.7 \pm 2.2$ & 0.200 \\
C20:4n-6 & $8.9 \pm 1.9$ & $10.2 \pm 2.6$ & $0.023^{*}$ \\
C18:3n-3 & $0.09 \pm 0.04$ & $0.10 \pm 0.11$ & 0.342 \\
C20:5n-3 & $0.34 \pm 0.15$ & $0.42 \pm 0.11$ & $0.005^{*}$ \\
C22:6n-3 & $2.1 \pm 0.9$ & $2.6 \pm 0.9$ & $0.032^{*}$ \\
C20:3n-9 & $0.16 \pm 0.06$ & $0.16 \pm 0.03$ & 0.972 \\
C20:5n-3/C20:4n-6 & $0.038 \pm 0.013$ & $0.044 \pm 0.018$ & 0.409 \\
C20:3n-9/ C20:4n-6 & $0.018 \pm 0.007$ & $0.017 \pm 0.006$ & 0.272 \\
C22:6n-3/C20:4n-6 & $0.23 \pm 0.07$ & $0.25 \pm 0.05$ & 0.079 \\
\hline
\end{tabular}

Values are mean $\pm \mathrm{SD}$.

* Statistically significant.

variable independently associated with PPAR $\gamma 2$ genotype (OR $0.75,95 \%$ CI $0.61-0.90$ ).

Lastly, a correlation analysis showed that in the present study HOMA-IR was negatively associated with plasma n-3 LCPUFA ( $p=0.002), \mathrm{n}-6$ LCPUFA $(p=0.022)$, C20:4n-6 ( $p$ $=0.016)$ and $\mathrm{C} 22: 6 \mathrm{n}-3(P=0.012)$. Considering the FA composition of plasma phospholipids, HOMA-IR was negatively associated with n-3 LCPUFA ( $p=0.021), \mathrm{C} 22: 6 \mathrm{n}-3$ ( $p$ $=0.046)$ and $C 22: 6 n-3$ to $C 20: 4 n-6$ ratio $(p=0.029)$, and positively with the $n-6 / n-3$ LCPUFA ratio $(p=0.003)$.

\section{DISCUSSION}

This study examined the association of plasma fatty acids with obesity and insulin resistance in normolipidemic obese children focusing on the differences between carriers of the more common PPAR- $\gamma 2$ Pro/Pro genotype and those carrying the possibly protective Pro12Ala variant. Indeed, because obesity is one of the most important risk factors for cardiovascular diseases and type 2 diabetes, obese children, who are presumably at a higher risk, may be protected from these diseases by the phenotypic effect of the Ala allele on insulin resistance since the Pro12Ala polymorphism of PPAR- $\gamma 2$ gene is associated with greater insulin sensitivity $(14,15)$. Through the years the PPAR- $\gamma 2$ Pro12Ala polymorphism beneficially influences insulin resistance and its tracking from childhood to adulthood (31). Also the rates of skeletal muscle glucose uptake are higher in subjects carrying the Ala allele than in subjects carrying the Pro12Pro genotype, at least within the non-obese population (32). The question whether Pro12Ala polymorphism modulates the skeletal muscle glucose uptake differently in non-obese and obese subjects is still open.

Skeletal muscle is a major site of insulin action, and insulin sensitivity may be related to the fatty-acid composition of phospholipids within the muscle membranes. LCPUFAs, in- 
cluding docosahexaenoic acid (C22:6 n-3, [DHA]), are major components of cell membranes. Low levels of DHA and other LCPUFAs, including arachidonic acid (C20:4 n-6 [AA]), and a high n-6/n-3 PUFA ratio in skeletal muscle membrane phospholipids are associated with insulin resistance in human adults $(33,34)$.

In young infants, breast-feeding increases LCPUFA levels in skeletal muscle membranes and early development of relatively higher levels of LCPUFAs in skeletal muscle phospholipids, influenced by either the type of feeding and/or genetic-related pathways, is associated with lower fasting plasma glucose levels (35). Accordingly, early changes in skeletal muscle membrane phospholipid FA saturation index may play a role in the subsequent development of diseases associated with insulin resistance. The less unsaturated muscle membranes in children whose mothers have higher fasting insulin and triglyceride levels may as well reflect a genetic reluctance to incorporate PUFAs into membranes, thus predisposing them to insulin resistance syndromes (36).

Since hypercholesterolaemia and hypertriacylglycerolaemia might substantially figure in defining plasma total fatty acid profiles, we have selected obese children showing blood lipid levels within a range of normality to prevent misinterpretations of data on the individual fatty acid status. Indeed the normal distribution of fatty acids differs significantly in cholesterols esters and triacylglycerols from those seen in phospholipids or free FA (37). Moreover in our sample, obese children with different PPAR- $\gamma 2$ genotype were comparable for plasma total cholesterol and triacylglycerol values.

The results of the present study suggest a possible link and explanation of the pathways leading to increased insulin sensitivity in carriers of the Pro12Ala genotype. Obese children with the Pro12Ala variant exhibited lower values of fasting insulin and HOMA-IR. In our study the average difference in Ala carriers compared with non carriers was $28 \%$ for fasting insulin levels and 30\% for HOMA-IR. Other authors (15) found an average difference of around 15\% (fasting insulin) and 17\% (HOMA-IR). However, we would point out that in the population studied by Buzzetti et al.(15), the mean HOMA-IR was 5.3 and 4.4 in Pro/Pro and Ala carriers, compared with 4.2 and 3.0 found in the present study. Values found in Buzzetti et al. (15) were therefore around 26-46\% higher. Accordingly, the two populations might be not directly comparable for variables possibly related to insulin sensitivity. It may be not excluded that children with different insulin sensitivity might differently respond to the PPAR $\gamma 2$ genotype Pro12Ala variant for these variables.

Obese children with the Pro12Ala variant showed higher levels of the major PUFAs (n-3 PUFA, n-6 LCPUFA, n-3 LCPUFA, C20:4n-6, C20:5n-3 and C22:6n-3), in plasma phospholipids. Plasma phospholipids may reflect the FA composition of red blood cell lipids since they are in continuous exchange (38). In turn, red blood cell FA composition may reflect the FA (particularly PUFA) composition of muscle membrane phospholipids, and it has been shown that the type of feeding shows similar effect on the LCPUFA composition of erythrocytes and muscles (39). Therefore our findings are consistent with the previous data of a lower representation of
C-20 and C-22 LCPUFA in membrane muscles of subjects with increased insulin resistance $(33,35)$. Furthermore, the higher levels of C:20:3 n-9 in total plasma lipids of obese children with the Pro/Pro genotype might indicate a homeostatic mechanism leading to an increased synthesis of the endogenous LCPUFAs derived from the $\mathrm{n}-9$ series, in absence of the highly unsaturated n-6 and n-3 compounds (40).

Since no major dietary differences between Ala carriers and non-carriers have been found, it may be hypothesized that either a preferential channeling of dietary LCPUFAs and/or a major LCPUFA endogenous synthesis from the parental n-6 and n-3 precursors could take place in obese children with the Pro12Ala variant. Whichever the case, and given a major protection from complications in the carriers of the Ala allele $(14,15)$, raising levels of LCPUFAs in membranes of Pro/Pro genotype carriers might be beneficial for insulin sensitivity in obese children. Finally, a logistic regression model disclosed that a lower $n-6 / n-3$ PUFA ratio in plasma phospholipids might be an indicator through which Pro12Ala exerts its effects on HOMA-IR, more than individual LCPUFA concentrations.

A limitation of the present study is the unfeasibility to estimate the dietary intake of the single FAs and then to evaluate the correlations between plasma FA values and dietary FA intakes. Indeed, this would be an important analysis as plasma FA levels are, presumably, a function of dietary intake and biologic processing of fats.

In conclusion, within the limitations of the present study, obese children carriers of the Pro12Ala variant seem to be protected from metabolic complications by pathways leading to a major representation of C-20 plus C-22 n-6 and n-3 LCPUFA in plasma phospholipids, presumably affecting in a parallel fashion the muscle membrane composition. Large longitudinal studies need to clarify the relationship between plasma PUFA and metabolic conditions with respect to PPAR $\gamma 2$ genotype in obese children and to study the dietary modifications able to improve the metabolic conditions in the carriers of the most common Pro/Pro genotype.

\section{REFERENCES}

1. de Onis M, Blossner M 2000 Prevalence and trends of overweight among preschool children in developing countries. Am J Clin Nutr 72:1032-1039

2. Ebbeling CB, Pawlak DB, Ludwing DS 2002 Childhood obesity: public-health crisis, common sense cure. Lancet 360:473-482

3. DeFronzo RA, Bonadonna RC, Ferranini E 1992 Pathogenesis of NIDDM. A balanced overview. Diabetes Care 15:318-367

4. Sinha R, Fisch G, Teague B, Tamborlane WV, Banyas B, Allen K, Savoye M, Rieger V, Taksali S, Barbetta G, Sherwin RS, Caprio S 2002 Prevalence of impaired glucose tolerance among children and adolescents with marked obesity. N Engl J Med 346:802-810

5. Saltiel AR 2000 The molecular and physiological basis of insulin resistance: emerging implications for metabolic and cardiovascular disease. J Clin Invest 106:163-164

6. Spiegelman BM 1998 PPAR-gamma: adipogenic regulator and thiazolidinedione receptor. Diabetes 47:507-514

7. Uauy R, Martinez JI, Rojas CV 2000 Molecular nutrition, role of the PPAR system in lipidic metabolism and its importance in obesity and diabetes mellitus. Rev Med Chil 128:437-446

8. Willson TM, Lambert MH, Kliewer SA 2001 Peroxisome proliferator-activated receptor gamma and metabolic disease. Annu Rev Biochem 70:341-367

9. Kota BP, Huang TH, Roufogalis BD 2005 An overwiew on biological mechanism of PPARs. Pharmacol Res 51:85-94

10. Stumvoll M, Haring H 2002 The peroxisome proliferator-activated receptor gamma2 Pro12Ala polymorphism. Diabetes 51:2341-2347 
11. Pihlajamaki J, Vanhala M, Vanhala P, Laakso M 2004 The Pro12Ala polymorphism of the PPAR gamma2 gene regulates weight from birth to adulthood. Obes Res 12:187-190

12. Altshuler D, Hirschhorn JN, Klannemark M, Lindgren CM, Vohl MC, Nemesh J, Lane CR, Schaffner SF, Bolk S, Brewer C, Tuomi T, Gaudet D, Hudson TJ, Daly M, Groop L, Lander ES 2000 The common PPARgamma Pro12Ala polymorphism is associated with decreased risk of type 2 diabetes. Nat Genet 26:76-80

13. Douglas JA, Erdos MT, Watanabe RM, Braun A, Johnston CL, Oeth P, Mohlke KL, Valle TT, Ehnholm C, Buchanan TA 2001 The peroxisome proliferator-activated receptor gamma2 Pro12Ala variant: association with type 2 diabetes and trait differences. Diabetes 50:886-890

14. Ghoussaini M, Meyre D, Lobbens S, Charpentier G, Clement K, Charles MA, Tauber M, Weill J, Froguel P 2005 Implication of the Pro12Ala polymorphism of the PPAR-gamma 2 gene in type 2 diabetes and obesity in the French population. BMC Med Genet 6:11

15. Buzzetti R, Petrone A, Caiazzo AM, Alemanno I, Zavarella S, Capizzi M, Mein CA, Osborn JA, Vania A, Di Mario U 2005 PPAR-gamma2 Pro12Ala variant is associated with greater insulin sensitivity in childhood obesity. Pediatr Res 57:138-140

16. Rhee EJ, Oh KW, Lee WY, Kim SY, Oh ES, Baek KH, Kang MI, Kim SW 2006 Effect of two common polymorphisms of peroxisome proliferator-activated receptor gamma gene on metabolic syndrome. Arch Med Res 37:86-94

17. Rodriguez-Cruz M, Tovar AR, del Prado M, Torres N 2005 Molecular mechanism of action and health benefits of polyunsaturated fatty acids. Rev Invest Clin 57:457-472

18. Storlien LH, Baur LA, Kriketos AD, Pan DA, Cooney GJ, Jenkins AB, Calvert GD, Campbell LV 1996 Dietary fats and insulin action. Diabetologia 39:621-631

19. Itoh T, Murota I, Yoshikai K, Yamada S, Yamamoto K 2006 Synthesis of docosahexanoic acid derivatives designed as novel PPARgamma agonists and antidiabetic agents. Bioorg Med Chem 14:98-108

20. Italian Society for Paediatric Nutrition (SINUPE) 2000 Guidelines for the prevention of atherosclerosis in pediatric age. IJP 26:13-28

21. Cole TJ, Bellizzi MC, Flegal KM, Dietz WH 2000 Establishing a standard definition for child overweight and obesity worldwide: international survey. BMJ 320:12401243

22. Cacciari E, Milani S, Balsamo A, Dammacco F, De Luca F, Chiarelli F, Pasquino AM, Tonini G, Vanelli M 2002 Italian cross-sectional growth charts for height, weight and BMI (6-20 y). Eur J Clin Nutr 56:171-180

23. Cole TJ 1990 The LMS method for constructing normalized growth standards. Eur J Clin Nutr 44:45-60

24. Matthews DR, Hosker JP, Rudenski AS, Naylor BA, Treacher DF, Turner RC 1985 Homeostasis model assessment: insulin resistance and beta-cell function from fasting plasma glucose and insulin concentrations in man. Diabetologia 28:412-419

25. Friedewald WT, Levy RI, Frederickson DS 1972 Estimation of the concentration of low density lipoprotein in plasma, without use of preparative ultracentrifuge. Clin Chem 18:499-502
26. Folch J, Lees M, SloaneStanley GH 1957 A simple method for isolation of total lipids from human tissues. J Biol Chem 226:497-509

27. Agostoni C, Trojan S, Bellù R, Riva E, Giovannini M 1995 Neurodevelopmental quotient of healthy term infants at 4 months and feeding practice: the role of long-chain polyunsaturated fatty acids. Pediatr Res 38:262-266

28. Saadeh S, Younossi ZM, Remer EM, Gramlich T, Ong JP, Hurley M, Mullen KD, Cooper JN, Sheridan MJ 2002 The utility of radiological imaging in nonalcoholic fatty liver disease. Gastroenterology 123:745-750

29. Block G, Hartman AM, Dresser CM, Carroll MD, Gannon J, Gardner L 1986 A data-based approach to diet questionnaire design and testing. Am J Epidemiol 124:453-469

30. Carnovale E, Marletta L 1997 Food composition tables. Istituto Nazionale della Nutrizione [Italian Institute of Nutrition], Milano, pp 16-80

31. Li S, Chen W, Srinivasan SR, Boerwinkle E, Berenson GS 2003 The peroxisome proliferator-activated receptor-gamma2 gene polymorphism (Pro12Ala) beneficially influences insulin resistance and its tracking from childhood to adulthood: the Bogalusa Heart Study. Diabetes 52:1265-1269

32. Vanttinen M, Nuutila P, Pihlajamaki J, Hallsten K, Virtanen KA, Lautamaki R, Peltoniemi P, Kemppainen J, Takala T, Viljanen AP, Knuuti J, Laakso M 2005 The effect of the Ala12 allele of the peroxisome proliferator-activated receptor-gamma2 gene on skeletal muscle glucose uptake depends on obesity: a positron emission tomography study. J Clin Endocrinol Metab 90:4249-4254

33. Borkman M, Storlien LH, Pan DA, Jenkins AB, Chisholm DJ, Campbell LV 1993 The relation between insulin sensitivity and the fatty-acid composition of skeletalmuscle phospholipids. N Engl J Med 328:238-244

34. Storlien LH, Pan DA, Kriketos AD, O'Connor ID, Cooney GJ, Jenkins AB, Baur LA 1996 Skeletal muscle membrane lipids and insulin resistance. Lipids 31:S261-S265

35. Baur LA, O'Connor J, Pan DA, Kriketos AD, Storlien LH 1998 The fatty acid composition of skeletal muscle membrane phospholipid: its relationship with the type of feeding and plasma glucose levels in young children. Metabolism 47:106112

36. Baur LA, O'Connor J, Pan DA, Storlien LH 1999 Relationships between maternal risk of insulin resistance and the child's muscle membrane fatty acid composition. Diabetes 48:112-116

37. Decsi T, Koletzko B 1994 Fatty acid composition of plasma lipid classes in healthy subjects from birth to young adulthood. Eur J Pediatr 153:520-525

38. Innis SM 1992 Plasma and red blood cell fatty acid values as indexes of essential fatty acids in the developing organs of infants fed with milk or formulas. J Pediatr 120:S78-S86

39. Baur LA, O'Connor J, Pan DA, Wu BJ, O'Connor MJ, Storlien LH 2000 Relationships between the fatty acid composition of muscle and erythrocyte membrane phospholipid in young children and the effect of type of infant feeding. Lipids 35:77-82

40. Holman RT 1964 Nutritional and metabolic interrelationships between fatty acids Fed Proc 23:1062-1067 